\title{
Changing Landscape of Clinical-Genomic Oncology Practice
}

\author{
Harsha Trivedi ${ }^{1}$, Heather M. Kling ${ }^{2}$, Tina Treece ${ }^{2}$, William Audeh ${ }^{2}$, Gordan Srkalovic ${ }^{1}$
}

${ }^{1}$ Clinical Trials and Precision Medicine Dept., Herbert Herman Cancer Center, Sparrow Hospital, Lansing System, MI, USA, ${ }^{2}$ Agendia, Inc., Irvine, CA, USA

Correspondence:

Gordan.Srkalovic@sparrow.org

Tel.: + 15173649400

Fax.: + 15173643687

Received: 25 September 2018

Accepted: 29 April 2019

Key Words: Genomics - Breast Cancer - Clinical Trials • Precision Medicine • Personalized Medicine.
The current paper discusses the use of genomics in the context of the changing landscape of clinical practice and modern medicine. Medical practice has shifted considerably over the past few decades, from empirical to evidence-based to personalized medicine, and the transition from reliance on observation to measureable parameters. Scientific innovation is required to collect an ever-increasing number and variety of data points and sophisticated analyses capable of distilling vast datasets into meaningful information. The next phase of innovation seeks to personalize disease management, in particular through genomics in oncology. With expanding use of genomics in medicine, and several initiatives collecting genomic data at the population level, education of patients and physicians is critical for data utility. By combining genomic and clinical data, bioinformatics approaches can be applied to developing individualized or targeted therapies. Breast cancer provides an example through which to understand the evolution of genomic data from pure science to clinical utility. From intrinsic subtype classification to development of multigene panels estimating recurrence risk, new studies, such as the FLEX trial, will expand to evaluate the whole transcriptome of tumours. This approach will enable discovery of novel gene signatures and ultimately pave the way toward a personalized approach to breast cancer management. Conclusion. Despite the potential for genomics to personalize treatments, a number of challenges remain to fully integrate these types of large datasets in a manner that provides clinicians and patients with meaningful, actionable information. However, if challenges are addressed, precision medicine has the capacity to transform patient care.

\section{Introduction}

For centuries, empirical evidence dominated the practice of medicine. Historically, clinical decision making was largely based on clinical experience, gained through trial and error. Despite the long history of research in medicine, approaches to incorporating the knowledge gained from basic and clinical research into the practice of medicine were generally non-standardized and subjective until recently. The utilization of re- search evidence in diagnosing and treating patients was determined by the individual physician, and, if incorporated, would have been alongside clinical experience and personal beliefs.

The use of evidence-based methods for both teaching and practicing medicine is founded in clinical epidemiology, and chronologically follows just behind evidence-based policies and guidelines, first published by David Eddy in a series of papers in the Journal of the American Medical 
Association (1-4). It concurrently de-emphasizes intuition and unsystematic clinical experience as rationale for clinical decision making (5). A shift toward evidence-based medicine has gained attention over the past two decades (6), seeking to integrate clinical expertise, the patient's personal preferences and the best available evidence in making health care decisions. Now an umbrella term "evidence-based medicine" captures both population- and individual-level decisions, this practice emphasizes the importance of incorporating evidence from formalized research into clinical decision making.

Evidence-based medicine is not without shortcomings (7). Evidence used in practice has been collected from large cohorts of patients, from which data are summarized into an expected response of the average patient from a given population (8). Although far more informative and accurate than its predecessors of intuition and the "art of medicine", the unfortunate consequence of this approach is that outliers are not represented, and they may be unlikely to respond similarly to the average patient for any given treatment.

Precision or personalized medicine, in contrast, focuses on the individual and seeks to improve health outcomes by integrating a huge variety and number of data points, from genomics to environmental and lifestyle factors, in order to provide an individualized approach to health care. Despite a lofty set of long-term goals, including earlier detection and better monitoring of disease symptoms, prediction of disease in asymptomatic individuals, more accurate prediction of treatment responses, improved disease surveillance, and prevention of disease, when possible, precision medicine has its own set of challenges and limitations. Some of these include small sample sizes (" $n$ of 1" studies), the technological capability required to compile large datasets, such as gene expression data or full exome sequenc- ing, and the informatics needed to distil vast amounts of data into clinically useful measurements.

There are strengths and weaknesses to both of these approaches, and integration of the strengths of both toward 'evidencebased precision medicine' will be complimentary and provide the best possible treatment for patients. Nonetheless, there are challenges to the integration of these two approaches. These include revisions to medical education and training programs, including training additional experts in clinical bioinformatics to interpret the large data sets that will be generated, and education of clinical professionals to stay current with the ever changing body of knowledge and to address the increasingly multidisciplinary practice of medicine. However, the benefits of successful integration of these approaches may be an ultimate shift in emphasis from reactive to proactive medicine, and a focus on prevention, rather than treatment, of disease (7).

Here, we will explore the challenges and progress of integrating genomics into clinical practice and several initiatives in various countries for large-scale genomic data collection at the population level. We will use breast cancer as an example through which to demonstrate the progression of genomic data into clinical utility and highlight how genomics and clinical data are being integrated for the discovery of new genomic signatures with the potential to provide individualized insights into disease management.

\section{Medical Science: From Observation to Measurement}

In addition to shifting paradigms to the practice of medicine, the science of medicine has transitioned from dependence on observation, empirical knowledge, and patient-reported symptoms to measurable parameters. This changing landscape has paralleled scientific and technological advances 
that continue to propel the field forward and allow for the collection of an ever-increasing number and variety of data points, enabling improved understanding of health and disease, as well as treatment options. For example, in a span of only a few decades, diagnostic tests have graduated from use of the microscope to visualize changes in cellularity that might indicate infection or inflammation to modern techniques, such as molecular testing, rapid detection and quantification of pathogens, automated blood chemistry panels, genetic and genomic testing, and sophisticated medical imaging. Furthermore, novel ways of measuring outcomes are moving from the idea of treating the disease to improving the patient's quality of life. Hospitals and healthcare systems are becoming more interested in patient-reported outcomes as a way of measuring progress and success. All of these datasets require sophisticated and sometimes complex analyses capable of distilling the mountain of information down to an actionable, or at least measurable, outcome.

\section{Advances in Science and Technology}

Advances in technology are making it possible to diagnose diseases, including cancer, earlier than ever before. For example, improvements in radiology and medical imaging have led to an uptick in early breast cancer diagnoses (9). Cancer Research UK reports that $31 \%$ of female invasive breast cancer cases in England are now detected by screening (10). Women diagnosed by this method are overwhelmingly Stage I-II, and with early treatment have observed steadily improving breast cancer survival since the 1990s (11). Despite these statistics, the American Medical Association (AMA) recommendation of regular mammographic screening for all women starting at age 40 has become controversial in the last decade. In November 2009, the US Preventative Ser- vices Task Force (USPSTF) recommended that screening mammograms should start at age 50 instead of 40 for women of average risk (12). The rationale was that although screen detection has improved the diagnosis of breast cancer, the rate of false-positive results was more common in women aged 40 49 , resulting in unintended consequences such as psychological harm and unnecessary imaging tests and biopsies in women without cancer. The Breast Cancer Surveillance Consortium recently evaluated 1,682,504 digital screening mammograms from 2007 to 2013 and confirmed that to be true (13). They determined that the while screen detection has increased the rate at which abnormal findings (AIR) are identified, since switching from film to digital images $(10.0 \%$ in 2008 to $11.6 \%$, Table 3 ), the proportion of these cases correctly detecting invasive cancer has declined from $90.3 \%$ specificity in 2008 to $88.9 \%$ in the current study.

Although many more women are now being treated for breast cancer, there has been no concomitant increase in survival benefit as a population (11). The USPSTF suggested that mammograms now detect small tumours, which previously would have gone undetected in the patient's lifetime, and unnecessary early treatment is pervading. In a pre-screening era these tumours, even untreated, may have posed minimal risk to the patient. Now the challenge is to identify which tumours are biologically low risk to appropriately spare patients from over-treatment, versus the tumours that are caught at a very early stage but are biologically high risk and require treatment.

In order to accurately distinguish high risk from low risk tumours, as well as to identify effective therapies, appropriate biological markers are needed. The same mutations may predominate in multiple cancers, and initiatives like The Cancer Genome Atlas (National Institutes of Health) are showing that there is substantial heterogeneity of ma- 
lignant drivers, even within the same 'type' of cancer (8), which is still classified according to its site of origin, as it has been historically. Heterogeneity of treatment responses can be found across all cancer types, but has the potential to be resolved with new biological marker discovery. Biological markers of cancer aggressiveness or treatment response have been identified in nearly every cancer and correlated to survival. Expanding availability of targeted therapies provide further opportunity to correlate genomic changes in the tumour with treatment responses, leading to improved understanding of targetable pathways involved in disease progression. The discovery of accurate biological markers has the potential to ultimately change the way cancer is described, diagnosed and treated, from a disease classified by its tissue of origin to an individualized classification based on a particular set of biological characteristics.

Although the development of novel and targeted therapeutics in some cancers has been made possible by the discovery of single gene mutations (genetics), other cancers rely on expression profiles that are composed of many genes (genomics). In breast cancer, advances have been driven by expression profiles more than single gene mutations. Although the heterogeneity and complexity of breast cancer has been recognized for some decades, the field shifted substantially with the publication of microarray-based gene expression profiles demonstrating this heterogeneity at the molecular level and the sub classification of breast cancer into molecular subtypes (14-16). These subtypes, luminal A, luminal B, normal breast-like, HER2, and basal-like $(15,17,18)$, have distinct clinical-pathological features, risk factors, responses to therapy, and clinical outcomes, demonstrating the clinical utility of molecular profiling. Following this discovery was the development of multigene panels to assess molecular characteristics of tumours and predict risk of recurrence in patients with early stage disease. Historically, clinical-pathological factors (patient age, tumour size, histopathologic features, lymph node involvement) have been used to estimate probability of breast cancer recurrence; however, there has been substantial interest in developing molecular assays that more accurately predict clinical outcome, thereby selecting patients who will most benefit from more aggressive therapies, while avoiding overtreatment in patients with comparatively low risk of distant recurrence. Presumably, selection of a combination of genes that provide information about a tumour's metastatic potential will most accurately predict distant recurrence in that patient. Several commercially-available molecular assays have been developed with the aim of providing this information (19). These assays use a variety of platforms, including reverse-transcriptase PCR (OncotypeDX, Breast Cancer Index, EndoPredict assays), Nanostring technology (Prosigna assay), and microarray technology (MammaPrint assay), for quantification of gene expression (19).

The first FDA-cleared multigene test, MammaPrint, uses a combined profile of 70 genes to assess metastatic potential of the tumour (20), thereby predicting a patient's risk of distant recurrence. The genes that comprise this signature function in proliferation, cell cycle dysregulation, invasion, angiogenesis, growth, and resistance to apoptosis (21). Genes were selected from the full transcriptomes of archived tumour specimens from patients without any systemic therapy (endocrine or chemotherapy) who either had a poor prognosis (distant metastasis within the first five years) or a good prognosis (no distant metastasis within the first five years). Using unsupervised hierarchical clustering, a signature of 70 genes was developed, representing the most differentially expressed genes between these two groups 
of patients (20). The 70-gene signature was further validated in subsequent studies (2226), and clinical utility was assessed in the prospective, randomized MINDACT trial, which compared outcomes following risk assessment by genomic or clinical parameters (27). The MINDACT trial demonstrated that patients at low genomic risk of recurrence by the 70 -gene signature could safely forego chemotherapy (27). The companion molecular subtyping assay to MammaPrint uses an 80-gene signature (BluePrint) to sub-categorize breast tumours as Luminal-, HER2-, or Basal-type (28-30). Compared with standard clinical subtyping, molecular assessment provides information about the functionality of the dominant molecular pathway in a tumour, and has been shown in studies to provide more accurate classification, as indicated by chemotherapy responses $(29,31)$. These assays demonstrate the clinical utility of genomics in breast cancer.

Biomarker discovery provides new opportunities for treatment personalization with advancing targeted therapeutics. Although using the immune system to treat cancer has a history dating from the late nineteenth century (32), cancer immunotherapy research and available therapies have exploded in recent years $(33,34)$. Immunotherapies, including checkpoint inhibitors, have shown remarkable success in generating durable responses in some patients, even in those for whom no other treatments were effective. However, these therapies have been underwhelming in others, and identification of biomarkers that accurately predict which patients will benefit from particular therapies will be crucial to ensuring more widespread success of these agents. Like other targeted therapies, immunotherapy is not a 'one size fits all' approach to cancer treatment. Although treatment success rates are higher in some cancers than others, immune checkpoint inhibitors are also not cancer site-specific. A variety of factors likely contribute to its success or failure, and understanding these factors will enable improved patient selection. The first FDA approval of an immune checkpoint inhibitor, pembrolizumab (Keytruda), which targets programmed death protein 1 (PD-1) on the surface of immune cells, thereby improving immune responses, was for patients with advanced melanoma. Since then, however, this inhibitor has been approved for use in at least 12 types of cancer and in patients with a common biomarker (tumours identified as microsatellite instability-high or mismatch repair deficient), irrespective of the cancer's site of origin (https://www.fda.gov/newsevents/newsroom/pressannouncements/ ucm560167.htm). With improved accuracy of biological markers and patient selection, it is possible that the success of immunotherapies will be expanded into cancers for which these treatment regimens have previously had limited success, including breast cancer $(35,36)$. It is likely that identifying the most predictive biomarkers or combination of biomarkers will require integration of tumour biology and host immune factors, merging genomics and immunology.

\section{Innovation in Data Capture and Analysis}

Integration of datasets consisting of imaging, molecular, genetic/genomic, cellular, organismal, environmental, family history and lifestyle data will be required in order to truly personalize medicine. However, learning and integrating the ever-growing mass of scientific discoveries and synthesizing it into an actionable recommendation will require immense data processing capacity. IBM Research announced in 2007 that the computer IBM Watson was taking on medical science. Watson would apply its DeepQA open-domain question answering technology to provide an evidence-based clinical decision support system. Watson uses natural 
language processing and machine learning to analyze unstructured information, overcoming the challenges with the structured data of traditional expert systems. It generates a list of possible questions and uses abductive reasoning to generate hypotheses and produce possible answers from the available information. In the healthcare setting, each potential answer then receives a confidence rating based on the supporting clinical and scientific evidence. If we can improve diagnostic accuracy, it could potentially be the most directly impactful step to improving our healthcare system.

Now that we are beginning to implement ways of integrating large and sometimes disparate pieces of information, the next challenge involves the production of content (data) necessary to generate meaningful conclusions and inform treatment recommendations. High throughput platforms, such as gene expression microarrays and next generation sequencing, produce thousands to millions of pieces of information per patient. Tied to clinical information, this big data provides the base from which questions can be asked and, one day, answered.

Several notable initiatives, including The Cancer Genome Atlas, the Cancer Moonshot, the 100,000 Genomes Project (United Kingdom) (37), the Sweden Cancerome Analysis Network - Breast (SCAN-B)[38], the NIH's All of Us research program, and France Genomic Medicine 2025, seek to enable scientific discovery through the integration of genomics and medicine on a large-scale, population-based level to improve collaboration, transparency and patient outcomes. France's Genomic Medicine 2025 aims to place France as a leader among major countries engaged in genomic medicine within the next 10 years. The first two genomic platforms were selected in 2017; 10 additional platforms are planned over the next five years. One will serve to meet the needs of patients suffering from cancer or rare diseases; the other is meant to begin sequencing genomes from the general population. Equipment and resources are planned to sequence the equivalent of 18,000 genomes per year. The 100,000 Genomes Project, in partnership with the National Health Service England, launched in late 2012, plans to sequence 100,000 genomes from NHS patients, targeting patients with rare diseases and those with cancer. The program aims to benefit patients and enable new scientific discovery, while also driving the UK genomics industry (37). The SCANB study was initiated in 2010 as a multicenter observational study to evaluate genomic profiles of breast cancer by whole transcriptome RNA-sequencing; by 2017, they had enrolled $>10,000$ patients and generated RNAseq data on $>7500$ specimens (38). The Cancer Genome Atlas (TCGA), a collaboration between the National Cancer Institute and the National Human Genome Research Institute to accelerate the understanding of the molecular basis of cancer through genome analysis technologies, collected data from 11,000 patients on 33 different tumor types during an 11 year period of study (https://cancergenome.nih.gov/abouttcga/ overview). President Obama announced the Cancer Moonshot Initiative in January 2016, acknowledging the need to accelerate discovery and encouraging change. The five strategic goals of the initiative are to catalyze new scientific breakthroughs, unleash the power of data, accelerate bringing new patent therapies to patients, strengthen prevention and diagnosis, and to improve patient access and care (https://www.cancersupportcommunity.org/sites/default/files/uploads/policy-and-advocacy/article/cancer_ moonshot_report_final.pdf). Notably, Vice President Joe Biden emphasized the need to engage patients as partners in research. The "All of us" research program was announced in 2016 by NIH to advance precision medicine. It has a goal to enroll 1 million or more 
volunteers from diverse backgrounds and lay the scientific foundation for a new era of personalized health care. Data will be collected from participants by surveys, their electronic records, and some participants will provide urine and blood samples. Data will be analyzed to learn about the health disparities and different health conditions.

\section{New Technologies Require Education}

Patients are already exhibiting a growing desire to participate in their own health/disease management, as well as to connect with others afflicted by the same disease or condition. The internet and modern social media tools provide endless resources to patients and their families, changing the dynamic of the patient-physician relationships. More information can be a double-edged sword, since there is just as much misinformation as reliable, verifiable information. It is now the job of health professionals and care givers to not only disseminate information, but to also contextualize and curate information given to them by patients. All of these new technologies require education, and despite the unprecedented wealth of information provided in the current era of genomics in medicine, many physicians do not have the training/expertise to interpret results from the deluge of genetic/genomic tests performed (39).

Through clinical trials, physicians and patients can work together to advance medical science. The traditional view of clinical trials is that a study is designed to answer a single question, usually in reference to a new drug compared to the current standard of care. Of late, the failure of trial after trial to produce a positive result has magnified some major flaws of traditional trial design, namely that trials take too long to show efficacy. Although counter-intuitive, it actually becomes even harder to show efficacy in disease states in which treatments are currently very effective. Adaptive trial designs are pushing the envelope by moving patients in and out of different treatment arms based on their response (40). Unlike the traditional model, patients who do not benefit from an experimental treatment are quickly offered alternatives. In savvy adaptive trials, clinical and genomic data from responders are used to then identify other patients with similar characteristics for future randomizations in order to optimize the chance of finding the right patient for the right therapeutic. The goal of the trial has now changed from examining how will a patient respond to an experimental therapeutic, to identifying who will most likely benefit from an experimental therapeutic. By linking genomics and clinical data we can apply bioinformatics approaches to developing individualized or targeted treatments, using novel genomic signature and biomarkers to identify patients who will respond, or just as importantly patients who will not respond.

\section{Linking Genomics and Clinical Data: the FLEX Study}

Advances in technology have enabled this paradigm shift in medicine, recognizing the importance of converging clinical factors and genomic pathway dependencies. Likewise, the ability to electronically capture clinical data efficiently has opened the door to novel trial designs that are accelerating the rate of insight discovery and hypothesis testing. For example, testing for the 70-gene MammaPrint (MP) signature has become standard of care in many early stage breast cancers, but largely unrealized is that MP is still tested on a microarray platform, similar to how it was developed. This has allowed for the untested genes that reside on the microarray platform, but are outside of the MP algorithm, to be tested against clinical outcomes at a costeffective rate, allowing the scale of research to not be limited by traditional trial funding. 
This scalable model is the foundation of the FLEX Registry protocol.

FLEX (MammaPrint, BluePrint and Fullgenome Data Linked with Clinical Data to Evaluate New Gene Expression Profiles: An Adaptable Registry) is a large-scale, population based prospective registry, sponsored by Agendia, Inc. The study is open to Stage I, II or III breast cancer patients and began enrolling patients in 2017. The FLEX study matches full-genome expression data (Figure 1) with comprehensive clinical data on patients enrolled in the study. Full genome profiling includes results from Agendia's two genomic signature assays, MammaPrint and
BluePrint. FLEX is patient-centered, agnostic to breast cancer subtype, management plan and treatment regimen. The study aims to collect 10 years of follow-up data on participating patients. By design, the study will not follow a defined endpoint, but uses a changing heuristic model driven by matching genomic data with comprehensive clinical data.

Unlike traditional trials, often designed without the downstream input of those who will later try to analyze the data (41), in FLEX, all participating investigators have the ability to propose concepts for investigation, which will shape the method and

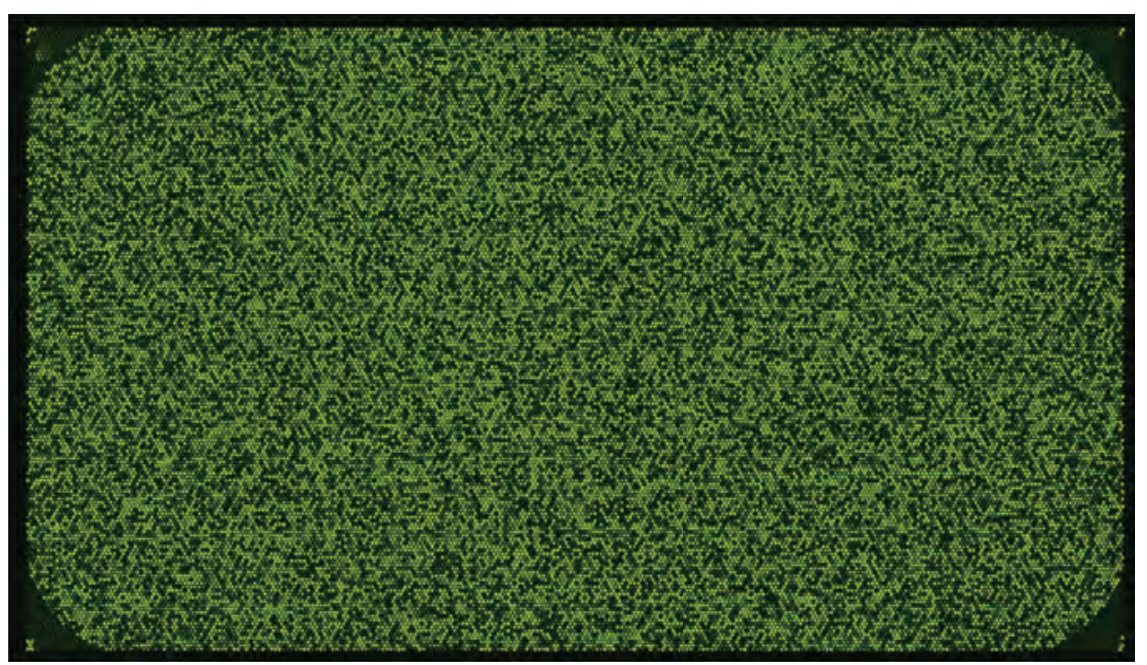

Figure 1. Representative pseudo-color image of microarray for full transcriptome.

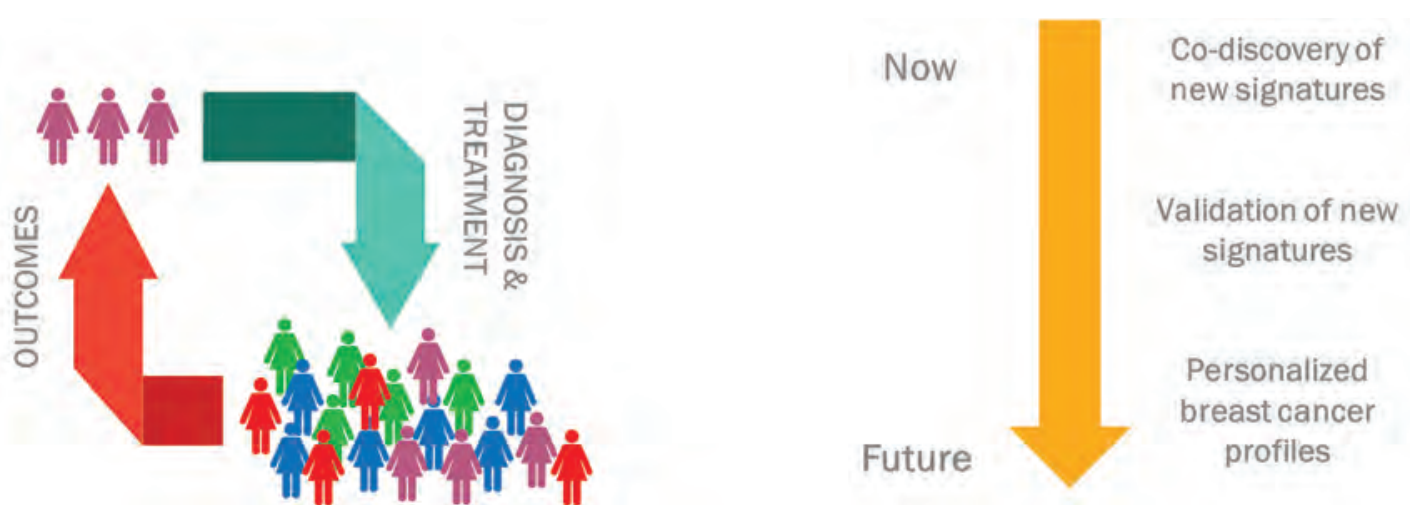

Figure 2. Model of the FLEX registry research cycle in which new gene signatures, discovered through retrospective analysis, can be validated in a larger population and used to create personalized breast cancer profiles in the future. 
structure of the clinical and genomic data collection of the trial, which will evolve over time (Figure 2). The scale of FLEX is also not limited to a defined enrollment target, which will allow obscure and yet-undefined clinical and genomic subsets to be adequately powered for study. This accelerated heuristic technique that FLEX employs has already produced compelling proof-of-concept arguments. Preliminary data analysis was performed on a subset of enrolled patients $(n=43)$ from a single location. A heatmap of quintile normalized gene intensities for top $25 \%$ of most variable genes from full genome transcriptome, across 44 samples (one patient had two submissions) clustered by Pearson correlation is shown in Figure 3 . Several clinical factors are included for comparison, demonstrating that the most differentially expressed genes in the breast tumors from these patients do not cluster by tumor grade, histopathologic tumor type, or lymph node involvement (Figure. 3). This method of clustering analysis applied over the nonlimited scale and proliferative trial objectives will reveal genomic signatures relevant to breast cancer management, as well as the landscape of clinical-genomic oncology practice.

\section{Summary/Conclusions}

Technological developments in genomic medicine are advancing at a breathtaking rate. The potential for precision medicine to match patients to gene-targeted therapy is a very intriguing promise, but simultaneously creates many challenges. One of the main challenges to implementing genomic medicine into clinical practice is conquering the knowledge gap arising from the extensive breadth and depth of data available

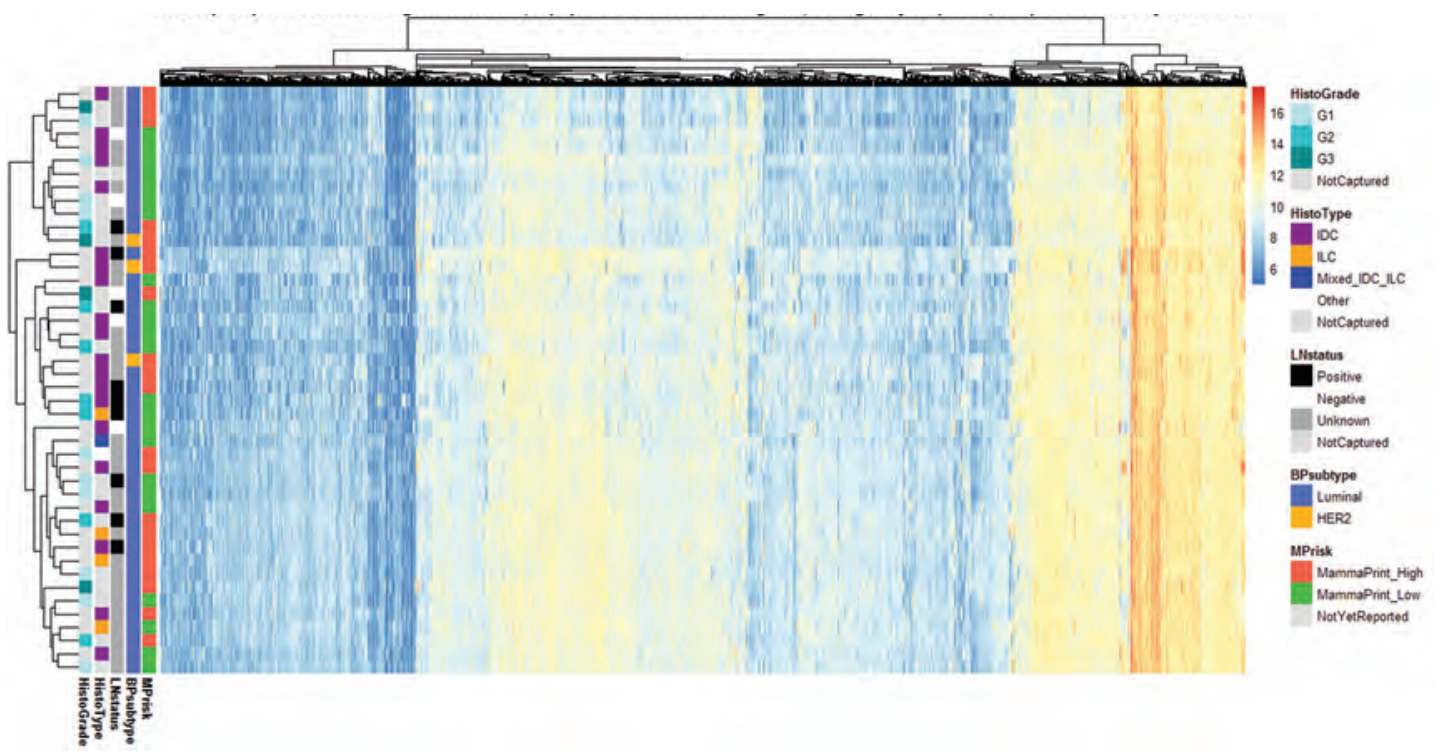

Figure 3. Heatmap of quantile normalized gene intensities for top $25 \%$ of most variable genes from full genome transcriptome, across 44 samples clustered by Pearson correlation. Visual representation of the relative amount of expression of all genes across the array for all tumor samples included in the analysis $(n=44)$. Expression of all genes in all tumor samples is relative to all other genes in order to visualize expression patterns among all tumor samples. Gene expression intensity is represented by colors, with blue indicating low expression and red indicating high expression. Each row includes the pattern of expression for all genes for a single tumor sample. Similar gene expression profiles are clustered more closely together, so that the most similar profiles are in closest proximity. Clinical characteristics (grade, tumor type, lymph node status, BluePrint subtype, MammaPrint result) associated with each tumor sample are indicated by the legend on the right and color-coded accordingly on the left side of the heatmap. 
through genomic testing. The complexity is then compounded by wide variations in the genomic landscape of tumors, both within and between cancer types. Most of our presently practicing oncologists were educated and trained in the era of empiric and "evidence-based" oncology and few have comprehensive training in techniques of rapidly advancing genomic medicine. Even fewer have the knowledge and skills necessary to embark on "data mining" of large datasets provided by gene expression profiling or full exome sequencing to distil vast amounts of data into clinically useful measurements.

In contrast, practicing oncologists have the clinical experience and expertise in patient care necessary to fill gaps that modern genomic analysis and massive data production create. Therefore, the future of oncology will rely on interdisciplinary collaboration between physicians, biologists, geneticists, bioinformatics specialists, and patients and their families. All participants will have to acquire and retain new knowledge and skills in order to provide the best individualized care to patients. To succeed, genomics must be included in the medical education of future physicians. This training process will undoubtedly be ongoing, facilitated and complicated by fast moving technological advances. The practice of medicine is changing along with advances that revise the science of medicine. In a new era of genomics and informatics, "one size fits all" treatments may soon be replaced with therapies that are truly personalized to an individual's unique combination of genes, environment, and lifestyle. Oncology is at the forefront of these advances; however, with all of the fervor these advances bring, there are challenges to be addressed in order to fully realize the potential that genomics and personalized medicine have in transforming patient care.

Authors' Contributions: Conception and design: TT, HMK, WA and GS; Acquisition, analysis and interpretation of data: HMK and TT; Drafting the article
HT, HMK and TT; Revising it critically for important intellectual content: HT, HMK, TT, WA and GS; Approved final version of the manuscript: HT, HMK, TT, WA and GS.

Conflict of Interest: HMK, TT and WA are employees of Agendia, Inc.

\section{References}

1. Eddy DM. Clinical decision making: from theory to practice. Anatomy of a decision. JAMA. 1990;263(3):441-3.

2. Eddy DM. Clinical decision making: from theory to practice. Connecting value and costs. Whom do we ask, and what do we ask them? JAMA. 1990;264(13):1737-9.

3. Eddy DM. Clinical decision making: from theory to practice. Resolving conflicts in practice policies. JAMA. 1990;264(3):389-91.

4. Eddy DM. Clinical decision making: from theory to practice. Practice policies --what are they? JAMA. 1990;263(6):877-8, 880.

5. Evidence-Based Medicine Working Group. Evidence-based medicine. A new approach to teaching the practice of medicine. JAMA. 1992;268(17):2420-5.

6. Masic I, Miokovic M, Muhamedagic B. Evidence based medicine - new approaches and challenges. Acta Inform Med. 2008;16(4):219-25.

7. Beckmann JS, Lew D. Reconciling evidence-based medicine and precision medicine in the era of big data: challenges and opportunities. Genome Med. 2016;8(1):134.

8. Collins FS. Exceptional opportunities in medical science: a view from the National Institutes of Health. JAMA. 2015;313(2):131-2.

9. Bleyer A, Welch HG. Effect of three decades of screening mammography on breast-cancer incidence. N Engl J Med. 2012;367(21):1998-2005.

10. Cancer Research UK. Breast cancer survival statistics; 2016. [cited 27 August 2018]. Available from: https://www.cancerresearchuk.org/health-professional/cancer-statistics/statistics-by-cancer-type/ breast-cancer/survival\#heading-Three.

11. Cossetti RJ, Tyldesley SK, Speers CH, Zheng Y, Gelmon KA. Comparison of breast cancer recurrence and outcome patterns between patients treated from 1986 to 1992 and from 2004 to 2008. J Clin Oncol. 2015;33(1):65-73.

12. US Preventive Services Task Force. Screening for breast cancer: U.S. Preventive Services Task Force recommendation statement. Ann Intern Med. 2009;151(10):716-26, W-236. 
13. Lehman CD, Arao RF, Sprague BL, Lee JM, Buist DS, Kerlikowske K, et al. National Performance Benchmarks for Modern Screening Digital Mammography: Update from the Breast Cancer Surveillance Consortium. Radiology. 2017;283(1):4958.

14. Perou CM, Sørlie T, Eisen MB, van de Rijn M, Jeffrey SS, Rees CA, et al. Molecular portraits of human breast tumours. Nature. 2000;406(6797):74752.

15. Sørlie T, Perou CM, Tibshirani R, Aas T, Geisler $\mathrm{S}$, Johnsen $\mathrm{H}$, et al. Gene expression patterns of breast carcinomas distinguish tumor subclasses with clinical implications. Proc Natl Acad Sci U S A. 2001;98(19):10869-74.

16. Sorlie T, Tibshirani R, Parker J, Hastie T, Marron JS, Nobel A, et al. Repeated observation of breast tumor subtypes in independent gene expression data sets. Proc Natl Acad Sci U S A. 2003;100(14):8418-23.

17. Perou CM, Parker JS, Prat A, Ellis MJ, Bernard PS. Clinical implementation of the intrinsic subtypes of breast cancer. Lancet Oncol. 2010;11(8):718-9; author reply 720-1.

18. Parker JS, Mullins M, Cheang MC, Leung S, Voduc D, Vickery T, et al. Supervised risk predictor of breast cancer based on intrinsic subtypes. J Clin Oncol. 2009;27(8):1160-7.

19. Kwa M, Makris A, Esteva FJ. Clinical utility of gene-expression signatures in early stage breast cancer. Nat Rev Clin Oncol. 2017;14(10):595-610.

20. van't Veer LJ, Dai H, van de Vijver MJ, He YD, Hart AA, Mao M, et al. Gene expression profiling predicts clinical outcome of breast cancer. Nature. 2002;415(6871):530-6.

21. Tian S, Roepman P, Van't Veer LJ, Bernards R, de Snoo F, Glas AM. Biological functions of the genes in the mammaprint breast cancer profile reflect the hallmarks of cancer. Biomark Insights. 2010;5:129-38.

22. Buyse M, Loi S, van't Veer L, Viale G, Delorenzi $\mathrm{M}$, Glas AM, et al. Validation and clinical utility of a 70-gene prognostic signature for women with node-negative breast cancer. J Natl Cancer Inst. 2006;98(17):1183-92.

23. Drukker CA, Bueno-de-Mesquita JM, Retèl VP, van Harten WH, van Tinteren $H$, Wesselinget J, et al. A prospective evaluation of a breast cancer prognosis signature in the observational RASTER study. Int J Cancer. 2013;133(4):929-36.

24. Drukker CA, van Tinteren $H$, Schmidt MK, Rutgers EJ, Bernards R, van de Vijver MJ, et al. Long-term impact of the 70-gene signature on breast cancer outcome. Breast Cancer Res Treat. 2014;143(3):587-92.

25. Knauer M, Mook S, Rutgers EJ, Bender RA, Hauptmann M, van de Vijver MJ, et al. The predictive value of the 70-gene signature for adjuvant chemotherapy in early breast cancer. Breast Cancer Res Treat. 2010;120(3):655-61.

26. van de Vijver MJ, He YD, van't Veer LJ, Dai H, Hart AA, Voskuil DW, et al. A gene-expression signature as a predictor of survival in breast cancer. N Engl J Med. 2002;347(25):1999-2009.

27. Cardoso F, van't Veer LJ, Bogaerts J, Slaets L, Viale G, Delaloge S, et al. 70-Gene Signature as an Aid to Treatment Decisions in Early-Stage Breast Cancer. N Engl J Med. 2016;375(8):717-29.

28. Glück S, de Snoo F, Peeters J, Stork-Sloots L, Somlo G. Molecular subtyping of early-stage breast cancer identifies a group of patients who do not benefit from neoadjuvant chemotherapy. Breast Cancer Res Treat. 2013;139(3):759-67.

29. Whitworth P, Stork-Sloots L, de Snoo FA, Richards P, Rotkis M, Beatty J, et al. Chemosensitivity predicted by BluePrint 80-gene functional subtype and MammaPrint in the Prospective Neoadjuvant Breast Registry Symphony Trial (NBRST). Ann Surg Oncol. 2014;21(10):3261-7.

30. Krijgsman O, Roepman P, Zwart W, Carroll JS, Tian S, de Snoo FA, et al. A diagnostic gene profile for molecular subtyping of breast cancer associated with treatment response. Breast Cancer Res Treat. 2012;133(1):37-47.

31. Whitworth P, Beitsch P, Mislowsky A, Pellicane JV, Nash C, Murray M, et al. Chemosensitivity and Endocrine Sensitivity in Clinical Luminal Breast Cancer Patients in the Prospective Neoadjuvant Breast Registry Symphony Trial (NBRST) Predicted by Molecular Subtyping. Ann Surg Oncol. 2017;24(3):669-75.

32. Coley WB. II. Contribution to the Knowledge of Sarcoma. Ann Surg. 1891;14(3):199-220.

33. Couzin-Frankel J. Breakthrough of the year 2013. Cancer immunotherapy. Science. 2013;342(6165): 1432-3.

34. Dizon DS, Krilov L, Cohen E, Gangadhar T, Ganz PA, Hensing TA, et al. Clinical Cancer Advances 2016: Annual Report on Progress Against Cancer From the American Society of Clinical Oncology. J Clin Oncol. 2016;34(9):987-1011.

35. Solinas C, Gombos A, Latifyan S, Piccart-Gebhart M, Kok M, Buisseret L. Targeting immune checkpoints in breast cancer: an update of early results. ESMO Open. 2017;2(5):e000255.

36. Nanda R, Liu MC, Yau C, Asare S, Hylton N, Van't Veer L, et al. Pembrolizumab plus standard neo- 
adjuvant therapy for high-risk breast cancer (BC): Results from I-SPY 2. J Clin Oncol. 2017;35(15 Suppl):Abstract 506.

37. Turnbull C, Scott RH, Thomas E, Jones L, Murugaesu N, Pretty FB, et al. The 100000 Genomes Project: bringing whole genome sequencing to the NHS. BMJ. 2018;361:k1687.

38. Rydén L, Loman N, Larsson C, Hegardt C, VallonChristersson J, Malmberg M, et al. Minimizing inequality in access to precision medicine in breast cancer by real-time population-based molecular analysis in the SCAN-B initiative. Br J Surg. 2018 Jan;105(2):e158-68.
39. Nelson EA, McGuire AL. The need for medical education reform: genomics and the changing nature of health information. Genome Med. 2010;2(3):18.

40. Bothwell LE, Avorn J, Khan NF, Kesselheim AS. Adaptive design clinical trials: a review of the literature and ClinicalTrials.gov. BMJ Open. 2018;8(2):e018320.

41. Azencott CA, Aittokallio T, Roy S; DREAM Idea Challenge Consortium, Norman T, Friend S, et al. The inconvenience of data of convenience: computational research beyond post-mortem analyses. Nat Methods. 2017;14(10):937-8. 